\title{
Flexible Consumers Reserving Electricity and Offering Profitable Downward Regulation
}

\author{
Nicolas Höning and Han La Poutré
}

\begin{abstract}
Previous work on demand response in smart grids considers dynamic real-time prices, but has so far neglected to consider how consumers can also be involved in planning ahead, both for scheduling of consumption and reserving their ability to regulate downward during balancing. This work models a flexible consumer in a novel two-settlement electricity auction. The consumer buys electricity on an ahead market and offers downward regulation on the balancing market. Bidding in twosettlement procedures is a hard problem and the use case of a flexible consumer is a novel problem setting. This poses a challenge for the design of smart ICT that automates bidding in smart grid operations. In a decision-theoretic model that takes the viewpoint of a flexible consumer, we implement a novel, unified format that simplifies computation of bids and can make the guarantee that offering downward regulation increases overall utility. Simulations show that the unified format, when compared to a benchmark format with two independent bids, attains the same utility under a wide range of market conditions despite its simplicity of use. Furthermore, it ensures that the consumer's offer for downward regulation is indeed executed with the $U N I$ format, which is often not the case with the benchmark format.
\end{abstract}

Index Terms-markets, balancing, flexibility, demand side management, downward regulation

\section{INTRODUCTION}

Electricity markets in smart grids are populated by two groups of actors: Those that pose the challenge to balance supply and demand on short notice (by deviating from their scheduled consumption or generation) and those who can react to it. Up to now, the latter group traditionally consisted mostly of generators that can ramp up quickly and few large consumers who are able to regulate downwards.

The introduction of new technologies, appearing on both the supply and the demand side, changes the nature of the balancing challenge and makes it more relevant to the smart grid community. First, renewable energy supply, which is of intermittent (not perfectly predictable) nature, is becoming more widespread and mature. Furthermore, more and more energy demand at lower levels of our electricity systems is becoming electrified. Electric vehicles (EVs) for transportation and heat pumps for heating are becoming part of our daily lives. Electricity storage technologies are pursued to alleviate the expected dynamics in energy systems, but will be very expensive in the foreseeable future.

This work is sponsored by Agentschap NL, a research funding agency of the Dutch ministry of economic affairs, via the project IDeaNeD in the IOPEMVT program.

N. Höning and H. La Poutré are with the CWI Amsterdam, The Netherlands (email: nicolas@cwi.nl). H. La Poutré is also with the department of computer science at Utrecht University, The Netherlands.
A significant part of the demand capacity on lower parts of the grid will (to some extent) be flexible on short notice, e.g. because it operates batteries, heating and cooling systems or EVs. It will also be controllable by smart ICT. Thus, many more market actors can soon contribute to resolving imbalance, and demand response becomes a crucial ingredient in smart grids. This development can be of use in all kind of smart grid implementations, for example microgrids or electronic market places with many actors.

Many market mechanisms trade electricity ahead of time, and also reserve balancing power ahead of time. An ahead market increases the number of actors who can take part in bidding, and therefore lowers prices. And, because it ensures that enough balancing capacity is available, it lowers uncertainty. Planning ahead is also of great importance in smart grid settings, but previous work on demand response in smart grids has so far neglected to consider how consumers can also be involved in planning ahead, both for scheduling of consumption ahead of time and reserving their ability to regulate downward during balancing.

A first inspiration on how to model market mechanisms that combine scheduling and balancing for future smart grids comes from current wholesale markets. The so-called twosettlement procedure is being used in many power markets around the world (e.g. [1], [2], [3]). It trades a continuous good (electricity) in two different markets, each being a doublesided auction. The first settlement clears an "ahead market" (usually one day ahead of time, but shorter intervals are possible in more dynamic market settings, e.g. in smart grid settings). Bidders submit a bid to buy or sell some quantity definitively. In addition, flexible suppliers submit a bid to the ahead market for the provision of optional reserve electricity. The second settlement then clears a "balancing market", in which inflexible consumers can buy balancing electricity from these previously procured reserves on short notice.

Bidding in the two-settlement procedure is complex and it is hard to construct bids. We investigate the bid optimisation problem of a flexible consumer on an ahead market that is part of the two-settlement procedure. This consumer buys some quantity on the ahead market and, due to his flexibility in his scheduling, offers a part of this quantity as downward regulation capacity on the balancing market. This is a task we can expect to be performed by smart software agents who control flexible consumption devices.

This work is based on a novel bid format for this complex market design problem, proposed in [4], where the focus was put on the generator side. This format unifies bids to both markets, which simplifies the computation problem of 
bidders. This is useful in smart grids, where participants will increasingly be required to perform fast and efficient decisionmaking, in order to react with small delays. Furthermore, bid functions can be submitted to the balancing market, which increases efficiency of allocations. [4] also introduced a market clearing mechanism for this unified bid format. In a parallel companion work to this work (currently under review and more tailored to computer science), we model the decisiontheoretic problem of bid construction for a generator. In this paper, we deal with the novel use case of a flexible smart grid consumer whose downward regulation can be reserved in the ahead market. This use case adds the additional challenge that the consumer forfeits utility of bought electricity for the outlook of uncertain revenues on the balancing market.

This work advances the state of the art in the following way: First, we adapt the unified bid format that was proposed in [4] to be used by flexible consumer. We also describe a market model in which it is possible to study the consumer's decision problem under uncertainty about market outcomes. Second, when using the existing alternative of submitting two independent bids to the ahead- and balancing markets, a flexible consumer who offers downward regulation has no guarantee that the revenues on the balancing market will compensate the lost utility of the downward regulation. We show that the unified bid format can make this guarantee for the case that the bid represents marginal valuation (which is the profit-maximising strategy in competitive markets), because the consumer spends less money on the same amount of electricity and also makes profits from selling downward regulation. Finally, we show through parametrised MonteCarlo simulations that our unified format performs well, when compared to a benchmark format with two independent bids. It attains the same utility under a wide range of market conditions and ensures that the consumer's offer for downward regulation is indeed executed. This is often not the case with the benchmark format, in settings in which the consumer is very uncertain about market outcomes or his own valuation of electricity is much larger than the market's valuation.

\section{BACKGROUND}

Modern electricity markets are centralised multi-unit auctions, to which actors submit bids for buying or selling electricity. We will first discuss bid formats and then broaden the literature discussion towards market designs.

A very common (e.g. [5], [6], [7]) mathematical model of bids in electricity markets, a so-called supply function, is based on a quadratic representation of total production costs: $f(Q)=C Q+R Q^{n} \cdot Q$ denotes a quantity of electricity and $C \in \mathbb{R}$ and $R \in \mathbb{R}$ are coefficients. Also, $C>0, R>0$ and $n>1$. (to model a consumer in the ahead market, this work uses $\left.f(Q)=C Q-R Q^{n}\right)$. Klemperer and Meyer (1989) [5] describe how supply functions combine Cournot and Bertrand modelling approaches and are useful in multiunit auctions when market outcomes are uncertain. Supply functions are considered to increase competition [8]. Cain and Alvarado (2004) [9] argue that quadratic functions provide a reasonable trade-off between an approximation of real-world generation costs and favourable modelling properties, such as good performance and smooth state transition behaviour in calculus-based market clearing algorithms.

Concerning the level of detail that goes into a bid, there are two general approaches to design markets for electricity [8], [10]: Markets commonly known as "pools" (an approach more popular in the US, e.g. the markets operated by PJM and MISO [2]) require bidders to specify non-convex costs and constraints of their operation (e.g. startup costs), which are taken into account by the auctioneer, possibly through side payments. Markets commonly known as "exchanges" (an approach more popular in Europe, e.g. the APX or EEX) do not allow to include such convexities in bids ${ }^{1}$. Exchanges offer the advantage of low transaction costs: market clearing requires the satisfaction of fewer constraints and can use a fast convex solver. Furthermore, bids are easier to compute (which is important for automated bidding and market clearing in settings where many actors bid frequently, such as in smart grids). Finally, bidders in both pools and exchanges will try to maximise their profits by adapting their bids. For market designers, the analysis of bidding strategies is easier when bids are less detailed. In this work, we model an exchange market.

Concerning the general approach to market clearing, electricity markets have used uniform-price auctions (UPA) designs, in which all participants pay or earn the same unit price. The last decade has seen more markets designed as discriminatory pricing auctions (DPA)[11], in which unit prices may differ based on individual bids, for example the England and Wales wholesale electricity market in March 2001. Generally, UPA settings have been found to result in more efficient allocations, but DPA designs lower prices and market power, see for example Fabra et al. (2002) [12] and Damianov et al. (2010) [13]. This paper models a DPA design.

Finally, we turn to balancing markets and the two-settlement procedure described in Section I. Deregulated balancing markets are a relatively young phenomenon and they solve a problem unique to electricity markets. Therefore, several questions about how to allocate reserve quantities and prices are not yet settled. One important issue is how the market maker procures reserve energy. The required optimal quantity is currently chosen by static heuristics, for example based on the capacity of the largest power plant, by remaining capacity in definitely allocated generation facilities, or on a percentage of historical peak capacity in the market. This paper uses a version of the two-settlement procedure in which the market maker allocates reserve capacity relative to definitely allocated capacity. Another issue is whether power should only be paid for when it is used for balancing or if the market should pay for the availability of fast-startable reserve capacity, regardless of whether it is put to use. The market model in this work uses the former approach.

Although they are, at the time of consumption, delivered together as an indistinguishable product, definitive and reserve electricity are priced independently. An important question for the market design is when to submit the supply bids for

\footnotetext{
${ }^{1}$ One exception is if the exchange allows bids for blocks of periods, but this work looks at one-shot scenarios only.
} 
each type. In sequential approaches (submit bids for optional electricity after the market for definitive electricity has been cleared), re-commitments can lead to inefficient allocations through strategic bidding. Therefore, most scientific literature favours simultaneous approaches (submit at the same time).

However, a problem with simultaneous approaches is that a bid in one market cannot refer to outcomes in the other market. This can reduce the chances of reaching efficient allocations. Only few proposals to tackle this problem exist. For example, Virag et al. (2011) [14] propose an iterative market design, where in each round the market maker proposes two market prices and the market participants update the quantities they would sell or buy at those prices. This runs until conversion, but the runtime properties of this dynamic method are uncertain. The problem has also been discussed in Höning et al. (2011) [4], where a combined bid format is proposed for the two-settlement procedure and a preliminary market clearing mechanism was sketched as constraint satisfaction problem. We will analyse this combined format in this work, as described in Section I.

\section{MODEL}

We study the decision problem of a flexible consumer $c$, who aims at maximising his utility. We define $c$ 's utility as the sum of the surplus from buying electricity in the ahead market $A$ and the profits of selling downward regulation in the balancing market $B$. His task is to construct appropriate bids for this. In this section, we describe the general form of bids, markets $A$ and $B$ and $c$ 's bid optimisation problem.

\section{A. Mathematical representation of bids}

A very common mathematical model of convex bids for generation in electricity markets are so-called supply functions, which are based on a quadratic representation of total production costs (see Section II): $f(Q)=C Q+R Q^{n}$. The marginal cost function is $f^{\prime}$, the derivative of $f: f^{\prime}(Q)=$ $C+2 R Q$ (we assume $n=2$, e.g. as in [7]). Marginal cost functions are of special interest in economic theory, as they are the profit-maximising bid of a supplier in a perfectly competitive market [15]. To model the a consumer $c$ 's valuation of electricity, we assume a total valuation function and a marginal valuation function, given by

$$
\begin{aligned}
V_{c}(Q) & =v_{c} Q-\alpha_{c} Q^{n} \\
V_{c}^{\prime}(Q) & =v_{c}-2 \alpha_{c} Q
\end{aligned}
$$

where $v_{c}$ denotes the maximal marginal valuation and $2 \alpha_{c}$ denotes the slope of the marginal valuation function. The slope is negative and thus models diminishing marginal valuation: the value of each bought unit decreases with increasing quantity. A bid in our market model is a linear function, mapping unit prices $\rho$ to quantities. The demand bid for $c$ is based on the inverse derivative of $V_{c}: V_{c}^{\prime-1}(\rho)=-\frac{1}{2 \alpha_{c}}\left(\rho-v_{c}\right)$. In our model, $c$ can adapt $v_{c}$ towards a bid in order to optimise his utility. In a bid $b_{c}$, we denote the adapted $v_{c}$ as $v_{c}^{*}$ :

$$
b_{c}(\rho)=-\frac{1}{2 \alpha_{c}}\left(\rho-v_{c}^{*}\right)
$$

\section{B. The two markets}

Both markets $A$ and $B$ are populated by suppliers and flexible as well as inflexible consumers. We only model imbalance of the demand side - inflexible consumers underestimate their demand in market $A$ and need balancing power in market $B$, whereas flexible consumers do not require balancing power. They can possibly sell balancing power, acting as suppliers on market $B$. Both markets accept continuous quadratic functions as bids and clear with a discriminatory pricing auction.

Let the upper limit of total demanded quantity in the market be $Q^{U}$ (we assume supply is not limited). Before market $A$ is cleared, each inflexible consumer $i$ submits his ahead demand bid $b_{i}^{A}$ and all actors who are flexible and thus can supply balancing power (this includes suppliers and flexible consumers) submit their bids $b_{f}^{A}$ and $b_{f}^{B}$ for both markets simultaneously (all bids are formatted as described in Section III-A). We call the market maker $S O$ (System Operator). The $S O$ uses all bids $b_{i}^{A}$ and $b_{f}^{A}$ to clear market $A$ and, as a result, allocates one part of $Q^{U}$ to be sold by suppliers and bought by consumers, called $Q^{A}$. Another part, called $Q^{o p t}$, is allocated from suppliers and flexible consumers as an option on balancing electricity and is determined by the $S O\left(Q^{o p t}\right.$ is a ratio of $Q^{A}$ and exceeds the possible balancing demand, see Section III-Bc). Later, in market $B$, some part $Q^{B} \in\left[0, Q^{\text {opt }}\right]$ of this option might be executed: When the inflexible consumers announce their demand $\in\left[0, Q^{o p t}\right]$ in market $B$ via their bids $b_{i}^{B}$, all balancing supply bids $b_{f}^{B}$ are used to clear market $B$, allocating balancing power $Q^{B} \in\left[0, Q^{o p t}\right]$. We assume here that all suppliers and flexible consumers can ramp up or regulate down fast enough to supply up to $Q^{\text {opt }}$ for balancing, like e.g. with gas power plants, batteries or EVs.

a) Aggregation of other actors: This work studies the decision problem of one flexible consumer agent $c$ and aggregates all other market participants as functions. Following [15], let $D(\rho) \rightarrow \mathbb{R}$ be an aggregated demand function and $S(\rho) \rightarrow \mathbb{R}$ an aggregated supply function. We will use $D$, $S$ and their parameters with the superscripts ${ }^{A}$ for market $A$ and ${ }^{B}$ for market $B$. The subscript ${ }_{-c}$ denotes explicitly that the function does not include $c . D$ and $S$ for markets $A$ and $B$ are given by

$$
\begin{aligned}
D_{-c}^{A}(\rho) & :=\left[D_{\max }^{A}-\alpha^{A} \rho\right]_{\geq 0} \\
S^{A}(\rho) & :=\left[\beta^{A}\left(\rho-\rho_{\text {min }}^{A}\right)\right]_{\geq 0} \\
D^{B}(\rho) & :=\left[D_{\text {max }}^{B}-\alpha^{B} \rho\right]_{\geq 0} \\
S_{-c}^{B}(\rho) & :=\left[\beta^{B}\left(\rho-\rho_{\text {min }}^{B}\right)\right]_{\geq 0}
\end{aligned}
$$

where $[X]_{\geq 0}$ denotes the maximum of $X$ and 0 , $D_{\max }^{A}, D_{\max }^{B}$ denote the maximal demand, $\rho_{\min }^{A}, \rho_{\min }^{B}$ denote the minimal unit offer price and $\alpha^{A}, \alpha^{B}$ as well as $\beta^{A}, \beta^{B} \in$ $[0,1]$ are slope parameters.

Furthermore, $Q^{A}=Q_{c}^{A}+Q_{-c}^{A}$, where $Q_{c}^{A}$ is the amount $c$ buys in market $A$, and $Q_{-c}^{A}$ is the amount bought by all other consumers. Similarly, $Q^{B}=Q_{c}^{B}+Q_{-c}^{B}$, where $Q_{-c}^{B}$ is 
generated by suppliers in addition to what they sold in market $A$ (or by flexible consumer selling downward regulation), while $Q_{c}^{B}$ is a reduction in $c$ 's consumption sold as downward regulation ( $c$ actually consumes $Q_{c}^{A}-Q_{c}^{B}$ ). Finally, we denote with $Q_{c}^{o p t}$ the maximal reduction that $c$ offers and with $Q_{-c}^{o p t}=Q^{o p t}-Q_{c}^{o p t}$ the maximal balancing power other suppliers hold available.

Given aggregated demand and supply bids from all other market participants, we model the residual supply function $S_{\text {res }}^{A}$ that $c$ effectively faces in market $A$ and the residual demand function $D_{\text {res }}^{B}$ that $c$ effectively faces in market $B$ (see also Fig. 1). In economic theory, residual supply is the full market supply minus the quantity bought by other consumers at each unit price $\rho$ and residual demand is the full market demand minus the quantity supplied by other generators (or flexible consumers) at each unit price $\rho$ [15]:

$$
\begin{aligned}
S_{r e s}^{A}(\rho) & =S^{A}(\rho)-D_{-c}^{A}(\rho) \\
D_{r e s}^{B}(\rho) & =D^{B}(\rho)-S_{-c}^{B}(\rho)
\end{aligned}
$$

b) Market clearing: We now explain market clearing on market $B$, where $c$ is selling balancing power. For market $A$, where $c$ is buying, the same principle applies.

Given $c$ 's bid $b_{c}^{B}$, the quantity $Q_{c}^{B}$ that $c$ sells and the unit price $\rho_{c}^{B}$ that $c$ earns are found at the intersection of residual demand and $c$ 's bid, thus $D_{\text {res }}^{B}\left(\rho_{c}^{B}\right)=b_{c}^{B}\left(\rho_{c}^{B}\right)$. However, we also need to consider outcomes of this intersection that would lead to invalid quantities, i.e. quantities not $\in\left[0, Q_{c}^{\text {opt }}\right]$. This restriction is not explicit in $b_{c}^{B}$. First, if $D_{\text {res }}^{B}\left(\rho_{c}^{B}\right)<0, c$ sells nothing, as his bid $b_{c}^{B}$ was too expensive. Furthermore, $Q_{c}^{B}$ cannot exceed $Q_{c}^{o p t}$ (for $S_{-c}^{B}$, we assume that individual capacity constraints of the aggregated suppliers are not exceeded and thus not relevant for the market clearing). $c$ is willing to sell $Q_{c}^{o p t}$ for a price of at least $\rho_{c}^{o p t}$ (thus, $\left.b_{c}^{B}\left(\rho_{c}^{o p t}\right)=Q_{c}^{o p t}\right)$ and the $S O$ is willing to pay at most $\rho_{S O}^{B}$ (thus, $\left.D_{\text {res }}^{B}\left(\rho_{S O}^{B}\right)=Q_{c}^{\text {opt }}\right)$. If $b_{c}^{B}\left(\rho_{c}^{B}\right)>Q_{c}^{o p t}$, we use a discriminative (also called pay-as-bid) auction approach [11], such that $c$ will sell $Q_{c}^{o p t}$ at a unit price of $\rho_{c}^{o p t}$ if $\rho_{c}^{o p t} \leq \rho_{S O}^{B}$ or sell nothing otherwise.

From now on, we denote with $\rho_{c}^{A}$ and $\rho_{c}^{B}$ the unit prices that $c$ earns on markets $A$ and $B$, respectively. For $c$ 's competitors, we denote with $\rho_{-c}^{A}$ and $\rho_{-c}^{B}$ the prices they earn.

c) Market coupling: Two parameters of market $B$ are determined by the outcome of market $A$. First, we assume that the maximum demand in market $B$ is related to $Q_{-c}^{A}$ via a ratio $r_{m}$, such that $D_{\max }^{B}=\frac{r_{m} Q_{-c}^{A}}{1-r_{m}}$. The $S O$ can approximate the ratio $r_{m}$ by experience. We assume for simplicity of our mechanism that he approximates $r_{m}$ perfectly and allocates $Q^{o p t}=D_{\max }^{B}$. Likewise, we assume that the $S O$ uses $r_{m}$ also for $c$ individually ${ }^{2}$, so it always holds that $Q_{c}^{o p t}=r_{m} * Q_{c}^{A}$. All other consumers are inflexible and thus use $r_{m}=0$, or we assume they are flexible and their downward regulation offers are aggregated in $S_{-c}^{B}$. As we explained earlier, we assume in this work that all suppliers can supply without limit, so we do not set $r_{m}$ for suppliers or flexible consumers besides $c$.

\footnotetext{
${ }^{2}$ Of course, a more detailed model would assume that bidders prefer different values for $r_{m}$. We proposed a market clearing mechanism in which bidders can submit several bids with different values for $r$ in [4]
}

TABLE I

SUMMARY OF PARAMETERS ${ }^{3}$

\begin{tabular}{|c|l|}
\hline Parameter & Description \\
\hline \hline$v_{c}$ & maximal valuation of $c$ \\
\hline$\alpha_{c}$ & slope of $c$ 's valuation function \\
\hline$D_{\max }$ & maximal demand of demand functions $D_{-c}^{A}$ and $D^{B}$ \\
\hline$\alpha$ & slope of demand functions \\
\hline$\rho_{\min }$ & min. price of supply functions $S^{A}$ and $S_{-c}^{B}$ \\
\hline$\beta$ & slope of supply functions \\
\hline$k$ & noise parameter \\
\hline$r_{m}$ & $\begin{array}{l}\text { describes ratio between } Q^{A} \text { (all demand) and } D_{\max }^{B} \\
\text { (reserve demand) as well as between } Q_{c}^{A} \text { and } Q_{c}^{\text {opt }}\end{array}$ \\
\hline
\end{tabular}

Second, we assume that in $S_{-c}^{B}, \rho_{-c}^{A}$ is used as the minimum price in market $B$ (which is denoted by $\rho_{\text {min }}^{B}$, see (4)).

d) Uncertainty: $c$ approximates the residual functions $S_{r e s}^{A}$ and $D_{r e s}^{B}$ with some uncertainty. We model this by noise parameters $k^{A}$ and $k^{B}$, with which we multiply the minimal price of suppliers in $S^{A}$ and $S_{-c}^{B}$ (see (3) and (4)):

$$
\begin{aligned}
S^{A}\left(\rho, k^{A}\right) & =\beta^{A}\left(\rho-\rho_{\min }^{A} k^{A}\right) \\
S_{-c}^{B}\left(\rho, k^{B}\right) & =\beta^{B}\left(\rho-\rho_{\min }^{B} k^{B}\right)
\end{aligned}
$$

\section{The bid optimisation problem}

$c$ has two bids to construct (we call them $b_{c}^{A}$ and $b_{c}^{B}$ ), one for market $A$ and one for market $B$. We now consider the optimisation of bids in terms of the utility across all transactions for $c$. Overall, $c$ 's utility $U_{c}$ is the valuation of the electricity he can use for himself, minus the price he pays for his initial allocation in market $A$, plus revenues through downward regulation in market $B$ :

$$
U_{c}=\left(Q_{c}^{A}-Q_{c}^{B}\right) V_{c}\left(Q_{c}^{A}-Q_{c}^{B}\right)-Q_{c}^{A} \rho_{c}^{A}+Q_{c}^{B} \rho_{c}^{B}
$$

However, to be able to estimate the effects of bids in both markets separately, we define a surplus function for market $A$ and a profit function for market $B$. In market $A$, we consider $c$ 's valuation of consuming $Q_{c}^{A}$ and the costs of buying $Q_{c}^{A}$. In market $B$, we consider the reward for reducing demand and denote the costs of $c$ 's downward regulation by the (lost) utility of the last $Q_{c}^{B}$ units in $Q_{c}^{A}$ (via the function $V_{c}^{e}$, see (9)):

$$
\begin{gathered}
\operatorname{surplus}_{c}^{A}\left(b_{c}^{A}, k^{A}\right)=V_{c}\left(Q_{c}^{A}\right)-Q_{c}^{A} \rho_{c}^{A} \\
\operatorname{profits}_{c}^{B}\left(b_{c}^{B}, b_{c}^{A}, k^{B}\right)=Q_{c}^{B} \rho_{c}^{B}-V_{c}^{e}\left(Q_{c}^{B}, Q_{c}^{A}\right) \\
V_{c}^{e}\left(Q_{c}^{A}, Q_{c}^{B}\right)=V_{c}\left(Q_{c}^{A}\right)-V_{c}\left(Q_{c}^{A}-Q_{c}^{B}\right)
\end{gathered}
$$

where $Q_{c}^{A}$ and $\rho_{c}^{A}$, as well as $Q_{c}^{B}$ and $\rho_{c}^{B}$, are determined through market clearing (see Sections III-Bb and III-Bd), and thus $b_{c}^{A}$ and $k^{A}$, as well as $b_{c}^{B}$ and $k^{B}$, are implicit in the righthand formulae. $c$ 's maximal capacity in market $A$ is given by $Q_{c}^{U}$ and his maximal capacity in market $B$ is given by $Q_{c}^{\text {opt }}=\frac{r_{m} * Q_{c}^{A}}{1-r_{m}}$. Note that profits $s_{c}^{B}$ is coupled to the results of market $A$ (and thus needs to consider $b_{c}^{A}$ ), as $Q_{c}^{A}$ is used in $V_{c}^{e}$ as well as in the determination of $Q_{c}^{o p t}$.

\footnotetext{
${ }^{3}$ With the exception of $v_{c}, \alpha_{c}, r_{m}$ and $Q_{c}^{o p t}$, we use them with superscripts ${ }^{A}$ or ${ }^{B}$ to denote their usage in market $A$ or $B$, respectively.
} 
We now formulate the bid optimisation problem for $c$, in which $c$ maximises his overall utility. It considers limited ranges of noise parameters $k^{A}$ and $k^{B},\left[k_{\text {min }}^{A}, k_{\text {max }}^{A}\right]$ and $\left[k_{\text {min }}^{B}, k_{\text {max }}^{B}\right]$, respectively. For the likelihood of $k^{A}$ and $k^{B}$, let the two probability lookup functions be $\operatorname{prob}^{A}\left(k^{A}\right) \rightarrow[0,1]$ and $\operatorname{prob}^{B}\left(k^{B}\right) \rightarrow[0,1]$. For each possible outcome for bid $b_{c}^{A}, c$ considers all possible outcomes for bid $b_{c}^{B}$ :

$$
\begin{aligned}
& \arg \max _{b_{c}^{A}, b_{c}^{B}}\left[\int _ { k ^ { A } = k _ { \text { min } } ^ { A } } ^ { k _ { \text { max } } ^ { A } } \operatorname { p r o b } ^ { A } ( k ^ { A } ) * \left(\operatorname{surplus}_{c}^{A}\left(b_{c}^{A}, k^{A}\right)\right.\right. \\
& \left.\left.+\int_{k^{B}=k_{\text {min }}^{B}}^{k_{\text {max }}^{B}} \operatorname{prob}^{B}\left(k^{B}\right) * \operatorname{profits}_{c}^{B}\left(b_{c}^{B}, b_{c}^{A}, k^{B}\right) d k^{B}\right) d k^{A}\right]
\end{aligned}
$$

\section{BID FORMATS}

We define a benchmark format (which we call $B E N C H$ ), where $c$ submits two independent bids. $c$ submits a bid $b_{c}^{A}$ to market $A$, which is a demand function of the format $b_{c}^{A}(\rho)=-\frac{1}{2 \alpha_{c}} *\left(\rho-v_{c}^{*}\right)$ (see Section III-A). The bid $b_{c}^{B}$ to market $B$ is a constant price $\rho_{c}^{B *}$. The bid optimisation problem does not differ from (10). We mark parameters with * if $c$ can adapt them in the bid. The $B E N C H$ bid format is (for instance) similar to the market design of the US Midwest system operator [2], which also requires simultaneous submission of bids to day-ahead and balancing market clearing, where the former bid is non-linear and the latter a constant price per MW.

In our proposed unified bid format (which we call $U N I$ ), $c$ only needs to submit one bid $b_{c}(\rho)=-\frac{1}{2 \alpha_{c}}\left(\rho-v_{c}^{*}\right)$. Prices for both ahead- and balancing power will be found on this bid. First, the $S O$ clears market $A$, using $b_{c}$ as $b_{c}^{A}$. Given the outcome of market $A$ ( $c$ buys $Q_{c}^{A}$ at unit price $\rho_{c}^{A}$ ), the $S O$ then constructs bid $b_{c}^{B}$ from $b_{c}$ to submit to market $B$. Bid $b_{c}^{B}$ is based on $b_{c}$, with $v_{c}^{*}$ replaced by $\rho_{c}^{A}$ and the slope inverted. It is defined for the unit price $\rho_{c}^{B}$ in the range $\left[\rho_{c}^{A}, \rho_{\text {max }}^{B}\right]$, where $b_{c}\left(\rho_{\max }^{B}\right)=Q_{c}^{A}-Q_{c}^{\text {opt }}$. See $b_{c}^{B}(\rho)$ in (11) and Fig. 1 for illustration.

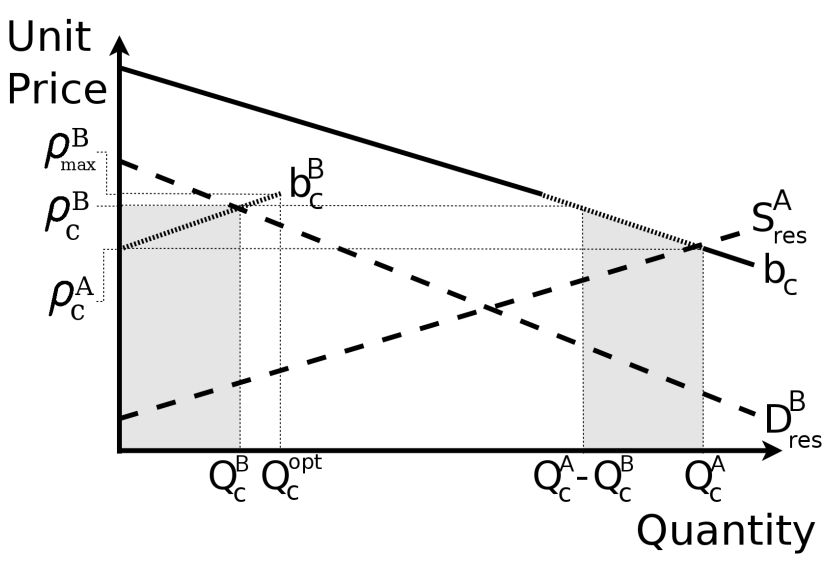

Fig. 1. UNI bid format with market outcomes. The dotted part of bid $b_{c}$ is translated into bid $b_{c}^{B} . Q_{c}^{A}$ and $Q_{c}^{B}$ are determined by intersection of $c$ 's bids with residual supply $S_{\text {res }}^{A}$ and demand $D_{\text {res }}^{B}$.

By design, the $U N I$ format has several advantages:
It reduces the complexity of c's optimisation problem in (10) from two to one dimension (only one bid $b_{c}$ is needed) and thus substantially simplifies the computation that a bidding agent has to perform (see (11)). Therefore, scheduling decisions can be made fast, which is an important feature for smart grid mechanisms.

$$
\begin{gathered}
\arg \max _{b_{c}}\left[\int _ { k ^ { A } = k _ { \text { min } } ^ { A } } ^ { k _ { \text { max } } ^ { A } } \operatorname { p r o b } ^ { A } ( k ^ { A } ) * \left(\operatorname{surplus}_{c}^{A}\left(b_{c}, k^{A}\right)\right.\right. \\
\left.\left.+\int_{k^{B}=k_{\text {min }}^{B}}^{k_{\text {max }}^{B}} \operatorname{prob}^{B}\left(k^{B}\right) * \operatorname{profits}_{c}^{B}\left(b_{c}^{B}, b_{c}, k^{B}\right) d k^{B}\right) d k^{A}\right] \\
\text { where } b_{c}^{B}(\rho)=\frac{1}{2 \alpha_{c}}\left(\rho-\rho_{c}^{A}\right)
\end{gathered}
$$

It defines a realistic price for balancing power: $\rho_{c}^{B} \in$ $\left[\rho_{c}^{A}, \rho_{\max }^{B}\right]$ relates to the actual reduction in (marginal) utility that $c$ suffers (according to his bid $b_{c}$ ) when buying $Q_{c}^{B}$ in market $A$, but not consuming it (because it is being sold on market $B$ ). Thus, the price for balancing power is related to the price on the ahead market. Varying the bid to exploit market opportunities is still possible for $c$, but speculating for profits on market $B$ is not possible in separation from the bid to market $A$. This is desirable, because two-settlement market designs generally aim at increasing the activity on and the importance of the ahead market stage. We will observe this effect in our simulations (see Section V). Because the slope of $b_{c}^{B}$ is positive, $\rho_{c}^{B} \geq \rho_{c}^{A}$, which reflects a natural relation between ahead- and balancing prices. For example, Oren (2000) [16] argues that balancing power is a good of higher economic quality than day-ahead procurement because of shorter delivery time and should be priced higher.

Furthermore, it guarantees $c$ that sales on market $B$ increase c's overall utility if $c$ bids his marginal valuation function as $b_{c}$. As a benchmark, we consider $c$ as an inflexible consumer, who buys $Q_{c}^{A}$ and does not selling anything on market $B$. His surplus $S U R_{c}^{\prime}$ is given by:

$$
S U R_{c}^{\prime}=\int_{Q=0}^{Q_{c}^{A}}\left(b_{c}(Q)-\rho_{c}^{A}\right) d Q
$$

Now we consider the case in which $c$ is flexible and active on market $B$, selling any $Q_{c}^{B} \in\left[0, Q_{c}^{\text {opt }}\right]$ at price $\rho_{c}^{B}$. Let us denote $c$ 's utility in this case by $U_{c}^{\prime}$. We calculate $U_{c}^{\prime}$ by subtracting from $S U R_{c}^{\prime}$ the loss of utility for $Q_{c}^{A}-Q_{c}^{B}$ and adding the revenues from selling $Q_{c}^{B}$. For illustration, Fig. 1 shows in a grey area both the lost utility (on the right) and the revenues from selling $Q_{c}^{B}$ (on the left). $U_{c}^{\prime}$ is given by:

$$
\begin{aligned}
U_{c}^{\prime} & =S U R_{c}^{\prime}-\int_{Q=Q_{c}^{A}-Q_{c}^{B}}^{Q_{c}^{A}} b_{c}(Q) d Q+Q_{c}^{B} \rho_{c}^{B} \\
& =S U R_{c}^{\prime}+\int_{Q=0}^{Q_{c}^{B}}\left(-b_{c}\left(Q+Q_{c}^{A}-Q_{c}^{B}\right)+\rho_{c}^{B}\right) d Q
\end{aligned}
$$

$U_{c}^{\prime}$ is guaranteed to be larger than $S U R_{c}^{\prime}$, if we can show that $b_{c}\left(Q+Q_{c}^{A}-Q_{c}^{B}\right) \leq \rho_{c}^{B}$ for all $Q \in\left[0, Q_{c}^{B}\right]$. This is the case, because $b_{c}\left(0+Q_{c}^{A}-Q_{c}^{B}\right)=\rho_{c}^{B}$ and the slope of $b_{c}^{B}$ is 
negative. Thus, we can conclude that $c$ will make profits by selling $Q_{c}^{B}$ when using the $U N I$ format.

Finally, we can now show that the UNI format guarantees that offering downward regulation increases c's utility, compared with an inflexible consumer that buys the same amount of electricity. As a benchmark, let us again assume that $c$ is inflexible and offers nothing to market $B$ (and thus $Q_{c}^{o p t}=0$ ). Here, we assume that $c$ buys exactly $Q_{c}^{A}-Q_{c}^{B}$ in market $A$. We denote the surplus for $c$ in this case by $S U R_{c}^{*}$, given by:

$$
S U R_{c}^{*}=\int_{Q=0}^{Q_{c}^{A}-Q_{c}^{B}}\left(b_{c}(Q)-\rho_{c}^{B}\right) d Q
$$

where in this example, $\rho_{c}^{B}=b_{c}\left(Q_{c}^{A}-Q_{c}^{B}\right)$ denotes the price $c$ pays for $Q_{c}^{A}-Q_{c}^{B}$ on market $A$ (refer also to Fig. 1).

Let us now consider that $c$ acts as a flexible consumer and offers $Q_{c}^{o p t}$ on market $B$. To make this case comparable to our benchmark case (which led to $S U R_{c}^{*}$ ), we assume that $c$ first buys $Q_{c}^{A}$ and then sells $Q_{c}^{B} \in\left[0, Q_{c}^{\text {opt }}\right]$. This leaves $c$ with $Q_{c}^{A}-Q_{c}^{B}$ for his own usage, just as in the benchmark case. We denote the utility $c$ has in this case with $U_{c}^{*}$. There are two differences in $U_{c}^{*}$ with respect to $S U R_{c}^{*}$ : First, $c$ pays a lower unit price for his consumption of $Q_{c}^{A}-Q_{c}^{B}$, namely $\rho_{c}^{A}$ instead of $\rho_{c}^{B}$, and if the price difference of $\rho_{c}^{B}-\rho_{c}^{A}$ is positive, $c$ 's utility will increase. Second, $c$ sells $Q_{c}^{B}$ instead of consuming it himself, and thus adds $U_{c}^{\prime}-S U R_{c}^{\prime}$ to his utility (see above). $U_{c}^{*}$ is given by:

$$
U_{c}^{*}=S U R_{c}^{*}+\left(\rho_{c}^{B}-\rho_{c}^{A}\right)\left(Q_{c}^{A}-Q_{c}^{B}\right)+U_{c}^{\prime}-S U R_{c}^{\prime}
$$

We have shown above that $U_{c}^{\prime}-S U R_{c}^{\prime}$ is positive. We now show that also $\left(\rho_{c}^{B}-\rho_{c}^{A}\right)\left(Q_{c}^{A}-Q_{c}^{B}\right)$ is positive, since $Q_{c}^{A} \geq Q_{c}^{B}$ and $\rho_{c}^{B} \geq \rho_{c}^{A}$ (because $\rho_{c}^{B}=b_{c}\left(Q_{c}^{A}-Q_{c}^{B}\right) \geq$ $\rho_{c}^{A}=b_{c}\left(Q_{c}^{A}\right)$, as the slope of $b_{c}$ is negative). Thus, offering reserves is profitable when $c$ bids his marginal utility function, by spending less money on the same amount of electricity and also making profits from selling downward regulation.

We note that, if the market setting is not competitive and thus consumers do not bid their marginal valuation functions (e.g., they bid a lower valuation function), the $U N I$ format does not guarantee profits for $c$ when offering downward regulation. The $S O$ can add an incentive $\iota$ when transforming $b_{c}$ to $b_{c}^{B}$, such that $b_{c}^{B}(\rho)=\frac{1}{2 \alpha_{c}}\left(\rho-\rho_{c}^{A}+\iota\right)$. He might choose to do so in a market mechanism as in [4], where bidders can choose the $r$ they want to bid on and the $S O$ finds he needs to incentivise bids with larger $r_{m}$ to allocate reserve capacity.

\section{Simulation EXPERIMENTS}

We construct two market scenarios and investigate several settings in each, using a systematic parameter analysis. In each setting, $c$ constructs bids for markets $A$ and $B$. We then investigate the resulting market outcome via Monte Carlo simulations.

\section{A. Market scenarios}

1) Oligopolistic market scenario: First, we define an oligopolistic market scenario, which could for instance resemble the situation in a microgrid. We model this scenario using realistic settings from a wholesale power market simulation study by Sun \& Tesfatsion (2007) [7]. Both microgrids and wholesale markets resemble oligopolistic market settings, because they are dominated by a small number of players. In [7], several generators and a generic buyer profile are described for 24 hours of a day on an electricity wholesale market. Note that, because we use settings from a wholesale market study, the prices in our model are in $\$ / M W h$ - but the general findings of this model can also hold for markets which trade $K W h$. In particular, the oligopolistic scenario corresponds to hour 8am in that study (we chose that hour as it is similar to most other hours and not an outlier).

We draw settings for the supply side by modeling an average generator $g$ from [7], with maximal production of $Q_{g}^{U}=300$, a minimum unit cost $C_{g}=18.8$ and a bid curve slope $R_{g}=0.008$. Furthermore, we assume that all generators in our model have the same slope in their production costs. [7] uses five generators in their model. Thus, we multiply the slope of $g$ 's marginal costs by five to get the slope of $S_{-c}$ : $\beta=\frac{5}{2 R_{g}}$. Finally, we assume that the minimal unit price of $S_{-c}$ is $10 \%$ higher than $g$ 's minimal unit costs: $\rho_{\min }^{A}=1.1 C_{g}$. As described in Section III-B, $\rho_{\min }^{B}$ is set to $\rho_{-g}^{A}$.

The sum of the demand of all buyers in [7] is 900 , or $3 Q_{g}^{U}$. We set $D_{\max }^{A}=3 Q_{g}^{U}\left(1-r_{m}\right)$. For the slope of the demand functions, we use a survey report [17] that aggregates several demand responsiveness studies. All studies in [17] measured the price elasticity of demand, which denotes the percentage change in quantity demanded in response to a one percent change in price. [17] distinguishes between "long-run" and "short-run" demand, where the latter allows less substitution of demanded power by any alternative, similar to the situation in a balancing market. The survey reports price elasticities between 0.7 and 2.1 for "long-run" scenarios (which we use for market $A$ ) and between 0.03 and 0.5 in "short-run" scenarios (which we use for market $B$ ). We take $\alpha^{A}=1.0$ and $\alpha^{B}=0.2$.

We model $c$ in the following way: His maximum capacity is $Q_{c}^{U}=Q_{g}^{U}=300$. For the slope of the valuation function of a flexible consumer, literature does not provide us with helpful pointers. For this work, we choose $\alpha_{c}=0.008$, mirroring the slope of the cost function of our average generator $g$. Finally, we aim at modeling $V_{c}$ such that $c$ 's valuation is close to the market valuation and set $v_{c}=\rho_{-c}^{A B} * 1.1 . \rho_{-c}^{A B}$ is the average price over markets $A$ and $B$ if $c$ is not present. The multiplication by 1.1 roughly compensates for the slope $\alpha_{c}$.

2) Competitive market scenario: We also design a second scenario (using the oligopolistic scenario as a starting point), in which we model two trends that are considered very important for smart grids. First, we make the scenario more competitive: we increase both the number of suppliers and demand responsiveness tenfold. Second, we model the trend towards increasing demand (e.g. through increasing market penetration of electric vehicles) by doubling the overall demand for electricity.

Table II lists all default settings for the two scenarios, where the parameters for market $B$ depend on the parameters of market $A$. Furthermore, we run simulations with $r_{m}=0.1$, which is a reserve level often in use today, as well as $r_{m}=0.3$, a setting that is not unrealistic in the market scenarios we can 
TABLE II

DEFAULT SETTINGS FOR MARKET PARAMETERS IN THE OLIGOPOLISTIC AND COMPETITIVE SCENARIO.

\begin{tabular}{|c|l|l|}
\hline Name & oligopolistic scenario & competitive scenario \\
\hline \hline$D_{\max }^{A}$ & $3 Q_{c}^{U}\left(1-r_{m}\right)$ & $6 Q_{c}^{U}\left(1-r_{m}\right)$ \\
\hline$\alpha^{A}$ & 1.0 & 10.0 \\
\hline$\rho_{\min }^{A}$ & $1.1 C_{g}$ & $1.1 C_{g}$ \\
\hline$\beta^{A}$ & $\frac{5}{2 R_{g}}$ & $\frac{50}{2 R_{g}}$ \\
\hline$D_{\max }^{B}$ & $\frac{r_{m} Q^{A}}{1-r_{m}}$ & $\frac{r_{m} Q^{A}}{1-r_{m}}$ \\
\hline$\alpha^{B}$ & $\frac{\alpha^{A}}{5}$ & $\frac{\alpha^{A}}{5}$ \\
\hline$\rho_{\min }^{B}$ & $\rho_{-c}^{A}$ & $\rho_{-c}^{A}$ \\
\hline$\beta^{B}$ & $\beta^{A}$ & $\beta^{A}$ \\
\hline
\end{tabular}

expect in the upcoming 10 years, at least for the generators that can offer significant reserve power (for example batteries or gas power plants).

\section{B. Method}

We evaluated both scenarios in multiple settings using a Monte-Carlo simulation, which we describe in more detail in this section. For each setting, $c$ constructs either two bids $b_{c}^{A}$ and $b_{c}^{B}$ (with the BENCH format) or one bid $b_{c}$ (with the $U N I$ format). First, $c$ performs a brute-force search on bid parameter settings: $c$ evaluates 100 evenlyspaced values for $v_{c}^{*} \in\left[\rho_{m i n}^{A}, v_{c}\right]$ and, with the $B E N C H$ format, also evaluates for each value of $v_{c}^{*} 100$ evenly-spaced values for $\rho_{c}^{B *} \in\left[\rho_{\text {min }}^{A}, \rho_{\text {max }}^{B}\right]$, where $S_{\text {res }}^{A}\left(\rho_{\text {min }}^{A}, 1\right)=0$ and $D_{\text {res }}^{B}\left(\rho_{\text {max }}^{B}, 1\right)=0$. Starting with the most promising point from the brute force evaluations, $c$ then applies a downhill simplex algorithm [18] to maximise his expected utility.

We sample the outcomes for each setting 100 times. Each sample uses a new pair of the noise parameters $k^{A}$ and $k^{B}$, generated by the Mersenne twister pseudo-random number generation algorithm. We assume $k^{A}$ and $k^{B}$ to be distributed normally and thus have to choose means and standard deviations in both markets $A$ and $B$. We set the means to 1 . We explain our choice for the standard deviation $s$ in each market using market $B$ as an example: We denote with $\rho_{\max }$ the maximal unit price in the market (such that $D_{\text {res }}^{B}\left(\rho_{\max }, 1\right)=0$ ). We then define $s$ such that $D_{c}\left(\rho_{\min }, 1+\phi s\right)=0$, where $\rho_{\text {min }}$ is the minimal price of $S_{-c}^{B}$ (see Section III-B) and $\phi$ is a scaling parameter normally set to 1 .

The difference of $\rho_{\max }-\rho_{\min }$ is dependent on the market setting and also differs in markets $A$ and $B$. Thus, the noise in the market is proportional to the maximal price variation in residual supply or demand. During the bid optimisation, $c$ considers values for $k \in\left[k_{\min }=1-3 s, k_{\max }=1+3 s\right]$.

To test for the stability of results, we varied several of the parameters. In both scenarios, we varied $\phi \in[0,3]$, $v_{c} \in\left[21,1.3 \rho_{-c}^{A B}\right], \alpha_{c} \in[0,0.01]$ and $r_{m} \in[0,0.3]$. In the oligopolistic scenario, we varied $\alpha^{A} \in[0,5], D_{\max }^{A} \in$ $\left[2 Q_{g}^{U}\left(1-r_{m}\right), 5 Q_{g}^{U}\left(1-r_{m}\right)\right]$. In the competitive scenario, we varied $\alpha^{A} \in[5,15], D_{\max }^{A} \in\left[4 Q_{g}^{U}\left(1-r_{m}\right), 10 Q_{g}^{U}\left(1-r_{m}\right)\right]$.

\section{Observations}

We begin by validating the market model for several important properties: $c$ 's overall utility is positive with both formats across all settings and, in comparison to the oligopolistic scenario, the competitive scenario has lower market prices and a lower utility for $c$. We now compare the two formats.

Observation 1: Both formats reach a comparable utility in a wide range of market conditions, but show different bidding behaviour. Under default conditions in both the oligopolistic and the competitive scenario, $c$ is more active on both markets when using the $U N I$ format: in market $A, c$ buys more $Q_{c}^{A}$ at a higher price $\rho_{c}^{A}$ and in market $B$, he sells more $Q_{c}^{B}$. However, the formats show no significant ${ }^{4}$ difference in the utility which $c$ achieves over all transactions. This observation is present in all our parametrised market settings (see Section V-B), with the only exceptions of very high values for $v_{c}$ and very low values for $\alpha_{c}$.

Observation 2: c's offer for downward regulation is indeed executed with the UNI format, but not with the BENCH format. With the $U N I$ format, $c$ consistently sells downward regulation across most market settings. However, when using the $B E N C H$ format, $c$ bids a price $\rho_{c}^{B *}$ that is too high in the given market setting, such that he sells no $Q_{c}^{B}$ or, when compared to the $U N I$ format, very little $Q_{c}^{B}$. The parameters that have the largest influence on $c$ 's activity on market $B$ are $\phi, v_{c}$ and $\alpha_{c}$. First, the more uncertain $c$ is about market outcomes (with increasing $\phi$, refer to Section V-B), the less downward regulation he sells when he uses the $B E N C H$ format (because he increases his bid on market $B$ ), while $c$ sells a constant amount when using the $U N I$ format. Second, in settings where $c$ 's valuation of electricity is higher than the market's valuation (with high values $v_{c}$ and $\alpha_{c}$, refer to Section V-A), $c$ does not sell any $Q_{c}^{B}$ with the $B E N C H$ format (because he bids too high prices in market $B$ ), while he sells stable amounts when using the $U N I$ format (see e.g. Fig. 2 for an example).

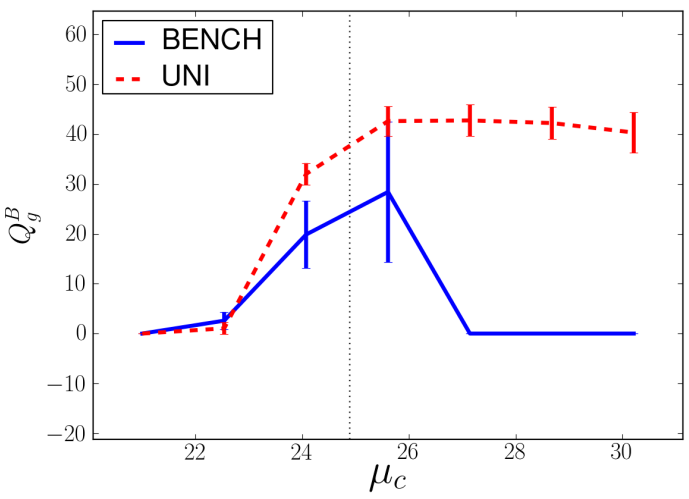

Fig. 2. $Q_{c}^{B}$, with one standard deviation, against increasing maximal utility of $c$ (oligopolistic scenario, $r_{m}=0.3$ ). The dotted line indicates the standard setting.

\section{Discussion of observations}

Let us first discuss observation 1, describing that despite different bidding behaviours, the two bid formats can reach a

\footnotetext{
${ }^{4}$ We performed Student's T-Tests and tested for $p \leq 0.01$.
} 
comparable utility. In most settings we studied, multiple nearoptimal combinations of quantities and prices exist. Though the $U N I$ format is less flexible than the $B E N C H$ format (because bids $b_{c}^{A}$ and $b_{c}^{B}$ are based on one bid $b_{c}$ ), it is likely to find a bid $b_{c}$ that realises one of them, as is evident in the good performance across all settings. This feature makes the $U N I$ format attractive to use for bidders.

Our second observation noted that with the $U N I$ format, $c$ will offer his downward regulation at acceptable prices, which makes the $U N I$ format attractive to the system designer. In particular, we saw that increasing noise leads $c$ with the $B E N C H$ format to raise prices on market $B$. This is because both the bid to market $B\left(\rho_{c}^{B *}\right)$ and the residual demand function for balancing power $\left(D_{\text {res }}^{B}\right)$ react only very little to changes in price: The former is a constant price, and the latter has a low slope. Thus, the intersection of both varies strongly along the quantity axis (which can also be seen in Fig. 2). Thus, the $B E N C H$ format increases the risk of having to reserve $Q_{c}^{o p t}$, but then selling too little $Q_{c}^{B}$ due to noise, which lowers overall utility. Therefore, $c$ raises his bid $b_{c}^{B}$ to cover the risk when uncertainty increases. This is not the case for the $U N I$ format, as here, $c$ submits a positively-sloped bid function to market $B$, which reduces variation along the quantity axis and leads to more precise predictions.

We also observed that with the $B E N C H$ format, $c$ increases prices on market $B$ to withhold $Q_{c}^{B}$ with large $v_{c}$ and low $\alpha_{c}$. The reason is that, when $c$ 's valuation of electricity is much higher than the market's valuation, it is optimal for $c$ to withhold $Q_{c}^{A}$ from market $B$. However, the $U N I$ format requires $c$ to offer balancing power at realistic prices, so in these market settings, $c$ cannot raise his bid to market $B$ to unacceptable prices.

\section{CONCLUSIONS AND FUTURE WORK}

In this work, we propose a novel approach to balancing in smart grids, which allows flexible consumers to offer downward regulation as reserve for the balancing market. We present a novel two-settlement electricity auction which uses a unified bid format (the $U N I$ format). Optimising bids with the $U N I$ format requires much less computation power when compared to a benchmark format with two independent bids, and therefore consumers can react fast. The auction guarantees that offering downward regulation is profitable to consumers. Furthermore, we show through parametrised Monte-Carlo simulations that the UNI format, despite its simplicity of use, attains the same utility as the benchmark format under a wide range of market conditions. Finally, it ensures that $c$ 's offer for downward regulation is indeed executed with the $U N I$ format, which is often not the case with the benchmark format.

The decision-theoretic approach taken in this work gives first insights into this complex problem setting. However, future work could further evaluate the $U N I$ format in more complex multiagent settings which run for multiple trade periods. It could also look into an extended market mechanism where individual bid slopes $\alpha_{c}$ and ratios $r_{c}$ per bidder are allowed (see also [4]).

\section{REFERENCES}

[1] Department Of Energy, "Impacts of the Federal Energy Regulatory Commission's Proposal for Standard Market Design," 2003.

[2] Midwest ISO, "Energy and Operating Reserve," Tech. Rep., 2010.

[3] TenneT, "The Imbalance Pricing System," Tech. Rep. June, 2005.

[4] N. Höning, H. Noot, and H. La Poutré, "Integrating power and reserve trade in electricity networks [extended abstract]," in Proceedings of the 10th International Conference on Autonomous Agents and Multiagent Systems (AAMAS), 2011, pp. 1293-1294.

[5] P. Klemperer and M. Meyer, "Supply function equilibria in oligopoly under uncertainty," Econometrica, vol. 57, no. 6, pp. 1243-1277, 1989.

[6] R. Baldick, "Electricity market equilibrium models: the effect of parametrization," IEEE Transactions on Power Systems, vol. 17, no. 4, pp. 1170-1176, Nov. 2002.

[7] J. Sun and L. Tesfatsion, "Dynamic Testing of Wholesale Power Market Designs: An Open-Source Agent-Based Framework," Computational Economics, vol. 30, no. 3, pp. 291-327, Aug. 2007.

[8] S. Stoft, Power Market Economics - Designing Markets For Electricity. Wiley-Interscience, 2002.

[9] M. Cain and F. Alvarado, "Implications of cost and bid format on electricity market studies: linear versus quadratic costs," in Large Engineering Systems Conference on Power Engineering, no. July, 2004.

[10] L. Meeus, "Is the prevailing wholesale market design in Europe and North America comparable?" Power Engineering Society General, pp. $1-5,2007$.

[11] V. Krishna, Auction Theory. San Diego: Academic Press, 2002.

[12] N. Fabra, N. Von Der Fehr, and D. Harbord, "Designing electricity auctions: uniform, discriminatory and vickrey," Universidad Carlos III de Madrid and University of Oslo, Tech. Rep., 2002.

[13] D. Damianov, J. Oechssler, and J. Becker, "Uniform vs. discriminatory auctions with variable supply-experimental evidence," Games and Economic Behavior, vol. 68, no. 1, pp. 60-76, 2010.

[14] A. Virag, A. Jokic, R. Hermans, and P. van Den Bosch, "Combined Bidding at Power and Ancillary Service Markets," in Proceedings of the 8th International Conference on the European Energy Market, Zagreb, 2011.

[15] J. Perloff, Microeconomics: Theory and Applications with Calculus. Addison Wesley, 2007.

[16] S. Oren, "Design of ancillary service markets," Proceedings of the 34th International Conference on System Sciences, 2000.

[17] R. Lafferty, D. Hunger, J. Ballard, G. Mahrenholz, D. Mead, and D. Bandera, "Demand responsiveness in electricity markets," Federal Energy Regulatory Commission, Tech. Rep., 2001.

[18] J. Nelder and R. Mead, "A simplex method for function minimization," The computer journal, vol. 7, no. 4, p. 308, 1965.

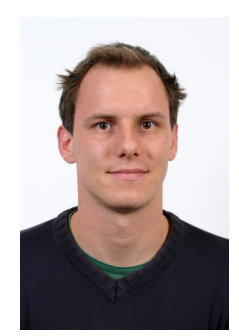

Nicolas Höning is a PhD student at CWI in the Multiagent and Adaptive Computation research group. His research examines market-based approaches to tackle the uncertainty for decentralised players in the domain of smart grids and energy systems. Nicolas holds a bachelor in cognitive science from the University of Osnabrück and a master in artifical intelligence from the Vrije Universiteit Amsterdam. Contact him at nicolas@cwi.nl.

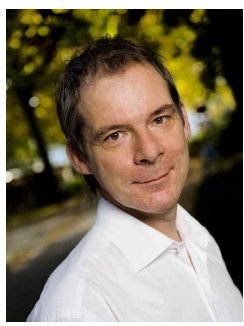

Han La Poutré is leader of the Multiagent and Adaptive Computation research group at CWI and professor at Utrecht University. His research interests include computational intelligence and multiagent systems, with applications to smart energy systems, logistics and e-business. La Poutré has a $\mathrm{PhD}$ in computer science from Utrecht University. He has been chair of the IEEE Technical Committee on Computational Finance \& Economics of the IEEE Computational Intelligence Society. Contact him at han.la.poutre@cwi.nl. 